\title{
A Study of the Factors Affecting Flange-Climb Derailment in Railway Vehicles
}

\author{
G. Diana, S. Bruni, E. Di Gialleonardo, R. Corradi and A. Facchinetti \\ Dipartimento di Meccanica \\ Politecnico di Milano \\ Italy
}

\begin{abstract}
Avoiding flange climb derailment is one main issue with ensuring the running safety of railway vehicles. This paper discusses the different causes that can lead to the derailment of a railway wheel, particularly in the light of different derailment criteria used by the standards or proposed by various researchers.

Furthermore the paper presents two case studies, one for a vehicle with solid axles and one for a bogie with independently rotating wheels, reporting a description of the derailment case and discussing the causes that led to derailment, by making combined use of measurements and numerical simulation.

Based on these exemplary cases, some conclusions are drawn concerning the validity of the derailment criteria presently used by the standards in force.
\end{abstract}

Keywords: flange climb derailment, Nadal's criterion, wheel-rail contact forces, running safety.

\section{Introduction}

Railways are known to be among the safest transportation mode. A survey conducted by the European Transport Safety Council reported the number of casualties per person and per travel hours in railways to be 14 times lower than for road transportation and 8 times lower than for air transportation. When the comparison is performed in terms of casualties per passengers and distance travelled the figure for railway is approximately 27 times lower than for road and similar to air, but the statistics do not include charter flights and private plane fatalities [1].

Nevertheless, rail accidents occasionally still happen and there is a need to further improve the levels of safety for this transportation mode. Excluding accidents occurring at level crossings and accidents to persons caused by rolling stock in motion (e.g. accidents to people working on the infrastructure), derailments represent a relatively frequent cause of accidents. For this reason, railway safety 
agencies at the national and supra-national level are continuously looking for ways to reduce the occurrence and impact of derailments, and to this same aim the European Union recently funded the research project D-RAIL [2].

Derailments in turn may be caused by several reasons, including failures occurring in the running gear, bad quality of the infrastructure, problems occurring at the wheel-rail interface and extreme environmental conditions. Aim of this paper is to discuss the mechanism of the so-called flange-climb derailment, i.e. a special but frequent case of derailment which happens when the wheel rolls over the top of rail and falls outside the track gauge. This mechanism can be triggered by a faulty condition of the running gear, by a defective track geometry (e.g. excessive track twist), by very high wheel-rail friction coefficient in short radius curves, or by a combination of the above. In the paper, the effect of some fundamental parameters affecting flange-climb derailment is discussed, also based on the examination of two case studies.

The paper is organised as follows: in Section 2 an analysis of the State-of-the-Art is reported, describing research work done so far to explain the mechanisms of flange-climb derailment and the criteria proposed to assess the safety against derailment of the rolling stock. In Sections 3 and 4 two different case studies are presented, reporting a description of the real derailment case, numerical simulations performed to analyse the causes, and the remedies proposed. Finally, in Section 5 some conclusions and recommendations for future research are drawn.

\section{Analysis of the State-of-the-Art}

\subsection{Derailment criteria}

The study of the wheel-rail contact conditions leading to flange-climb derailment of a railway wheelset has been the object of extensive research in the past and has led to the definition of different derailment criteria that can be used to assess running safety of railway vehicles. A survey of the safety assessment criteria in use in different world regions and countries can be found in [3].

Most of the safety criteria are based on setting a limit on the so-called derailment coefficient $\mathrm{Y} / \mathrm{Q}$, i.e. the ratio between the lateral $\mathrm{Y}$ and the vertical (normal to the top of rail plane) $\mathrm{Q}$ components of the contact forces acting on the flanging wheel. We use here the notation from the European standard EN14363, whereas in other regions such as the USA the lateral and vertical force components are often denoted by $\mathrm{L}$ and $\mathrm{V}$ and $\mathrm{L} / \mathrm{V}$ is used for the derailment coefficient.

The limit value for the derailment coefficient is often based on the famous formula due to Nadal [4]:

$$
\left(\frac{Y}{Q}\right)_{l}=\frac{\operatorname{tg} \gamma_{M A X}-\mu}{1+\mu \operatorname{tg} \gamma_{M A X}}
$$

with $\mu$ the wheel-rail friction coefficient and $\gamma_{M A X}$ the maximum contact angle which depends on the wheel and rail profiles. 
In general, the limit value expressed by Eq. (1) tends to be over-conservative, as it implies that the maximum creep force available at wheel-rail contact for a given normal force is entirely applied in transversal direction, producing the maximum possible wheel uplift effect $[5,6]$. In many cases, however, the uplift force can be lower due e.g. to small transversal creepage / spin, to the presence of longitudinal creep forces or, in the case of a solid wheelset, to saturation effects taking place on the other wheel.

Indeed, extensive research activities reported e.g. in [5-9] have shown both experimentally and by means of mathematical modelling and simulation that the limit value for the derailment coefficient is also affected by other parameters, such as the angle of attack of the wheel over the rail and the ratio between the vertical forces on the flanging and non-flanging wheels. Furthermore, this limit value also depends upon the magnitude of the longitudinal force acting at wheel-rail contact. To consider these effects, alternative derailment criteria have been proposed, taking into account the following parameters:

a. the sum of the Y/Q ratios on the flanging and non-flanging wheels [10];

b. the wheelset's angle of attack $[6,7,8,9,11]$;

c. the ratio of the longitudinal force over the vertical one, combined with the $\mathrm{Y} / \mathrm{Q}$ ratio [6].

It should be noted that the mechanism of wheel derailment is significantly different for a solid wheelset compared to an axle with independently rotating wheels. Hence, the derailment criterion best suited to describe the wheelset's proneness to derailment can be different depending on the specific design concept.

It is also worth pointing out that the derailment criteria introduced in ref.s [6-11] are all based on the consideration of the flange-climb process as a quasi-static one, whereas in many cases derailment occurs as the consequence of violent shocks produced by local track defects. More research might be needed to assess the validity of the above criteria in dynamic conditions and/or to propose new criteria considering in full the dynamics of the wheelset.

\subsection{Causes of flange-climb derailment}

Another important research stream is concerned with investigating the relationships existing between the vehicle's running conditions (including parameters such as speed, cant deficiency, track geometry, suspension parameters, rail and wheel defects) and the risk of derailment.

This is often investigated by means of multi-body simulation of the vehicle's running behaviour, but requires the use of accurate wheel-rail contact models for which a specific validation is advisable. In [12] some general guidelines and requirements for defining a suitable multi-body model of a railway vehicle are provided, while in [13] a state-of-the-art review of models for suspension components can be found. Ref.s [14, 15] provide examples of multi-body models and running dynamics analysis specifically addressing safety and derailment issues. 
In general, the following causes are identified as passible of producing flange-climb derailment in a vehicle.

\subsubsection{Sharp curves}

Sharp curves tend to produce large Y/Q values on the flanging wheel of the leading wheelset in a bogie, as the consequence of the creep forces generated on account of the wheelset's angle of attack. Additionally, the relatively large angle of attack itself promotes the derailment because, as show in the references introduced in Section 2.1, derailment may occur at lower $\mathrm{Y} / \mathrm{Q}$ ratios when the angle of attack is larger.

For a given curve radius, longer bogie wheelbase and higher primary yaw stiffness are responsible for larger angle of attack values and therefore negatively affect the risk of derailment. Cant deficiency generates an additional lateral force on the wheelset due to the effect of non-compensated centrifugal forces.

\subsubsection{Track twist}

Large values of track twist may arise in curve transitions due to the track cant gradients and/or may result from track irregularities. Excessive track twist can be dangerous as it causes the unloading of two opposite wheels in the bogie (e.g. front left and rear right) and a corresponding increase of load on the other two wheels. When track twist is such that the outer wheel in the leading wheelset is unloaded, this combines with the effect of creep forces that push the wheel into flange contact, so that very high values of the Y/Q ratio can be attained, possibly leading to the derailment of the wheel. In order to compensate track twist and reduce the risk for wheel unloading, articulated bogie frames are sometimes adopted, especially in trams and light rail vehicles.

\subsubsection{Vehicle dynamics and instability}

Derailment may arise from intense vehicle dynamics due to different causes: vehicle resonant response to track irregularities, lateral or hunting instability of the vehicle, localised defects in the track (cusps, joints, ...). In these cases, the derailment mechanism is eminently dynamic and a clear understanding of the effect of different parameters is still subject for further research.

A special case is represented by vehicles with independently rotating wheels. In these vehicles the self-centring effect is only provided by the gravitational stiffness implied by conicity and is much smaller than for a vehicle with solid axles. This implies a higher sensitivity of the vehicle to localised track irregularities [14].

Furthermore, longitudinal creep forces tend to be negligible in a pair of independently rotating wheels (apart from the effect of traction and braking), so that the transversal creep forces may reach the full saturation limit promoting the flange climb process.

Another special case is when large lateral forces are applied on the vehicle due to the effect of crosswind. In this case the vehicle initially undergoes an overturning process, which is then followed by derailment. 


\subsubsection{Faults in vehicle suspensions}

Faults in vehicle suspensions may lead to an unbalanced distribution of vehicle static loads. In this case, some wheels will be subject to a lower static load than in a normal operating condition, thereby resulting to be more prone to derailment.

Alternatively, a fault in the suspensions may lead to increased sensitivity of the vehicle to track twist. The typical example is a vehicle that sits on deflated air springs. The stiffness of the rubber bushings realising in this case the secondary suspension of the vehicle is much higher than the vertical stiffness of the inflated air spring, resulting in larger load transfer for the same amount of twist applied to the bogies. To ensure safe running of the vehicle in this condition, twist tests are performed also for the vehicle with deflated suspensions. A similar effect may take place when the vertical primary stiffness is increased due to a fault. In this case, the bogie becomes more sensitive to track twist.

\section{Case study A: vehicle with solid axles}

The first test case presented in this paper refers to a vehicle for urban transport. The research activity consisted of two steps. First, a mathematical model of the vehicle, validated by means of a first experimental campaign, was used to reproduce the conditions that led to the derailment of the vehicle under analysis, establishing the causes of the accident. Secondly, a more extensive experimental campaign was designed to verify and quantify the running safety margin of the vehicle, following a modification of the secondary suspension design.

As far as the first phase of the research activity is concerned, a mathematical model of the vehicle was developed to carry out a numerical simulation of the accident in order to investigate the possible causes.

Being interested in a low-frequency phenomenon (below $20 \mathrm{~Hz}$ ) a rigid body schematisation was used and the non-linearities due to the wheel-rail contact and the suspensions were fully accounted for.

The simulated track section consisted of a narrow curve (nominal radius equal to $250 \mathrm{~m}$ ). The track geometry was measured and considered as input for the simulation. Figure 1 reports the curvature and the superelevation of the track as a function of the longitudinal position.

It is clearly visible from the superelevation diagram (Figure 1 (b)) that almost no full curve section is present on the track, being the curve characterised mainly by the transitions. The curvature diagram (Figure 1 (a)) is more difficult to understand being not properly coherent with the superelevation. In fact, deviations from the ideal geometry are observed both in the amplitude and in the position of the transitions.

Figure 2 (a) shows the values of the vertical Q and lateral Y components of the contact force and the derailment coefficient $\mathrm{Y} / \mathrm{Q}$ as a function of the longitudinal position along the track. Results are reported for the derailing wheel only. 
(a)

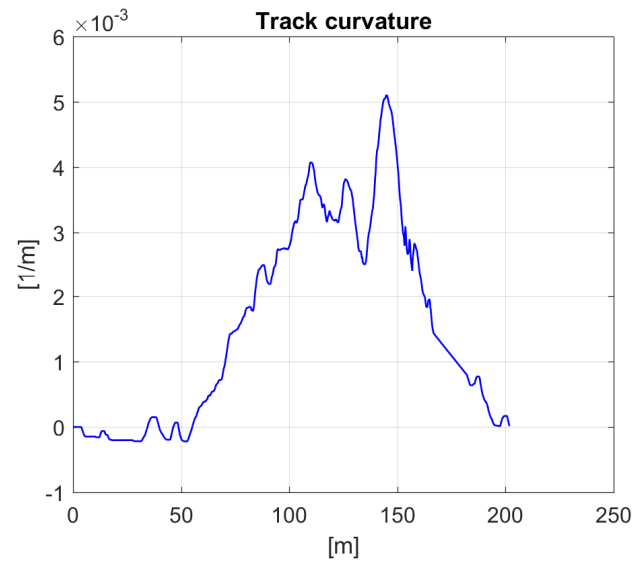

(b)

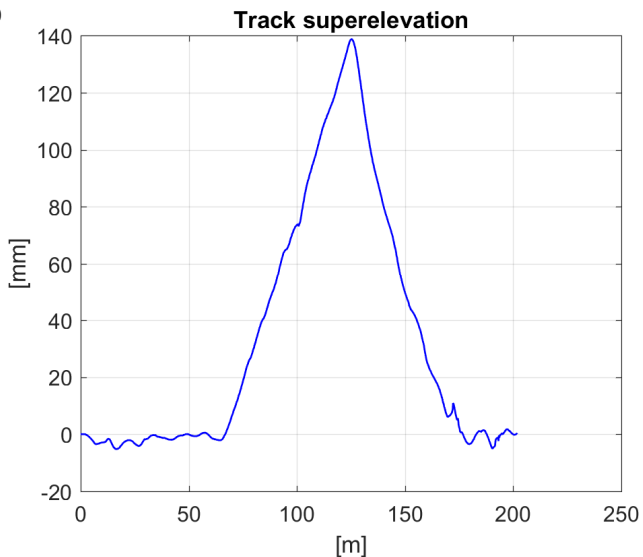

Figure 1: (a) track curvature and (b) track superelevation of the simulated curve.

(a)
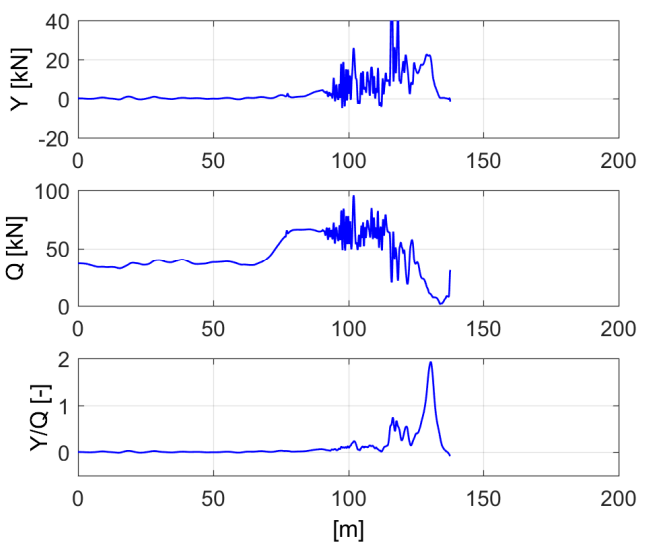

(b)

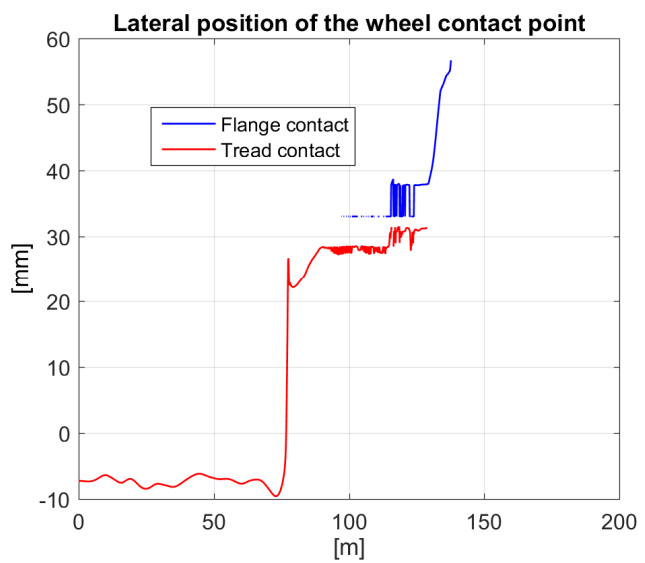

Figure 2: (a) Lateral force $Y$, vertical force $Q$ and derailment coefficient $Y / Q$ on the derailing wheel, (b) lateral position of the contact points on the wheel profile obtained by means of a numerical simulation.

The vehicle generates a guiding force approximately equal to $10 \mathrm{kN}$ during the first part of the entry transition curve (position around $70 \mathrm{~m}$ ); on the contrary, with reference to the force component $\mathrm{Q}$, a large reduction of the vertical force is observed in the first part of the exit transition curve (position $130 \mathrm{~m}$ approximately) leading to the almost complete unloading of the wheel. This unloading is mainly due to a large value of the track twist which, in degraded vehicle conditions, is not compensated in a satisfactory way by the suspension system. Therefore, the Y/Q ratio reaches values close to 2 and the wheel is lifted off the rail.

The derailing process is better described in Figure 2 (b) where the lateral position of the contact point on the wheel is shown. It can be observed that when the large wheel unloading occurs the contact on the tread (red line) is lost and the lateral position of the contact point on the flange (blue line) quickly increases up to values corresponding to the condition of contact between the external surface of the flange and the rail head. 
Thus, the numerical analysis suggests that the degraded condition of the suspension, associated with a large value of the track twist, caused the accident.

An experimental campaign was set-up in order to verify the effectiveness of a proposed modification of the suspension arrangement and to quantify the safety margin, even in degraded condition.

The bogie of a trailer vehicle (characterised by a lower axle-load with respect to a motor vehicle) was instrumented with different sensors. Additionally, one of the two wheelsets was instrumented using strain gauge bridges both on the axle and on the wheels to measure wheel-rail contact forces, as described in [16].

In this way, during on-track tests it was possible to measure the deformations of the axle in the measuring sections and to infer in real-time the wheel-rail contact forces.

The analysis of the experimental data provided in this section refers to a particular track section having a length equal to approximately $1.3 \mathrm{~km}$, that is characterised by a series of six narrow curves with maximum radius $500 \mathrm{~m}$. Table 1 specifies the position of the curves along the track together with their direction and the value of the radius.

\begin{tabular}{|c|c|c|c|c|}
\hline Curve n. & Initial position [km] & Final position [km] & Radius [m] & Direction \\
\hline 1 & 0.04 & 0.27 & 250 & left \\
\hline 2 & 0.34 & 0.57 & 300 & left \\
\hline 3 & 0.63 & 0.75 & 500 & right \\
\hline 4 & 0.77 & 0.95 & 500 & left \\
\hline 5 & 0.95 & 1.03 & 500 & right \\
\hline 6 & 1.10 & 1.21 & 250 & right \\
\hline
\end{tabular}

Table 1: list of the curves present in the analysed track section.

Figure 3 reports at the top the measured vertical and lateral forces on the right wheel of the leading axle, while at the bottom the corresponding unloading coefficient $\Delta \mathrm{Q} / \mathrm{Q}_{0}$ (defined as the wheel unload with respect to the static vertical value $\mathrm{Q}_{0}$ ) and derailment coefficient $\mathrm{Y} / \mathrm{Q}$ are shown.

Two different tests are compared, performed at the same speed, but with the vehicle in two different configurations in terms of sensitivity to the track twist. Time histories of the quantities referred to the vehicle with the higher sensitivity are plotted using a blue line, while a red line is used for the vehicle with the lower sensitivity. The limit values defined according to the standard Fiche UIC 518 [17] are indicated using a thick black line.

The analysis of the contact force components shows that the lateral force is very similar whereas important differences are found on the vertical force. The vehicle with the higher sensitivity to the track twist experiences larger variations of the vertical component of the contact force in the curve transitions. In particular considering a left-hand curve the right wheel shows a larger unloading during the exit transition, while an opposite situation is obtained considering a right-hand curve. 
Thus, the vehicle with the higher sensitivity to track twist exhibits larger variations of the unloading coefficient in the transitions, without exceeding the limit value equal to 0.6. Analogously the derailment coefficient $Y / Q$ is larger in curve exit when the curve is left handed so that the right wheel is the outer one. In this case, in the exit transition of the first curve the Y/Q value exceeds the limit fixed at 0.8 , however it does not exceed Nadal's limit, which, considering the wheel profile used, is equal to 0.95 if the coefficient of fiction is 0.5 .

(a)

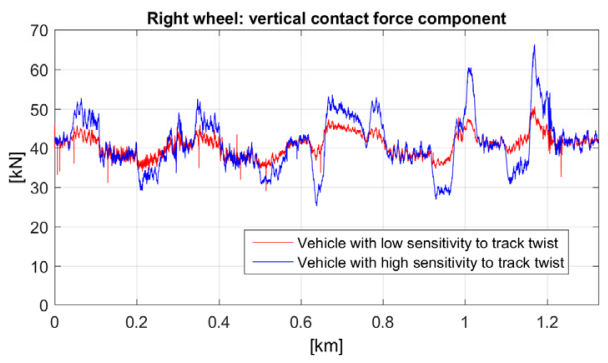

(c)

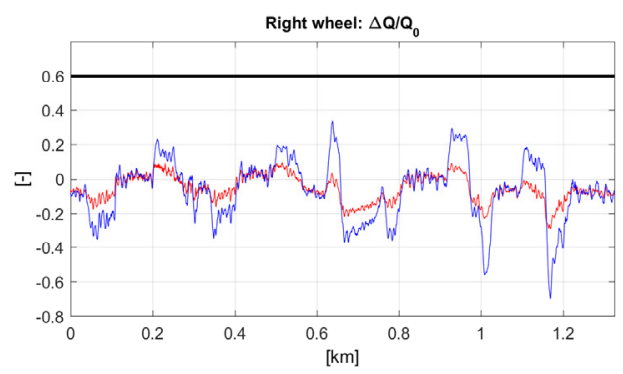

(b)

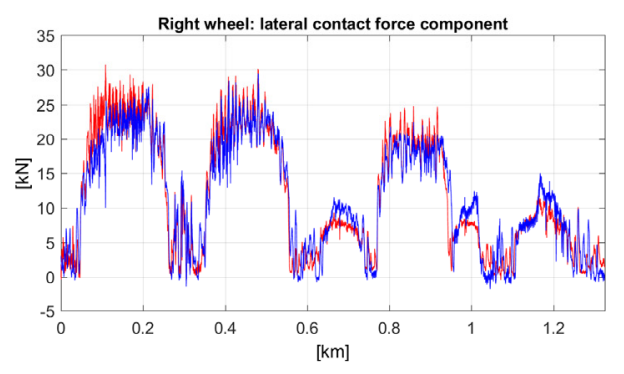

(d)

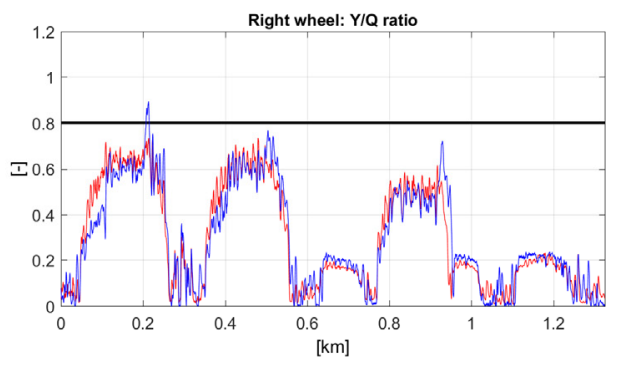

Figure 3: (a) vertical force $\mathrm{Q}$, (b) lateral force $\mathrm{Y}$, (c) unloading coefficient $\Delta \mathrm{Q} / \mathrm{Q}_{0}$ and (d) derailment coefficient $\mathrm{Y} / \mathrm{Q}$ on the considered wheel of the instrumented wheelset for two different vehicle configurations.

As one last example, the case of a track section where very large values of the derailment coefficient were measured on the vehicle having the higher sensitivity to the track twist without resulting in a derailment, is reported. The Y/Q coefficient and the unloading coefficient $\Delta \mathrm{Q} / \mathrm{Q}_{0}$ are shown in Figure 4 . It is observed that the $\mathrm{Y} / \mathrm{Q}$ coefficient (Figure 4 (a)) during the first curve exit reaches a value of 1.7, much larger than the Nadal's limit which, as already said, is equal to 0.95 in this case. As already discussed, this extreme value is ascribed to the unloading of the wheel due to the track twist during curve exit, as shown by the unloading coefficient reported in Figure 4 (b). The combination between the small value of the vertical force $\mathrm{Q}$ and the large value of the lateral force $\mathrm{Y}$, produces the very large value of the derailment coefficient. 
(a)

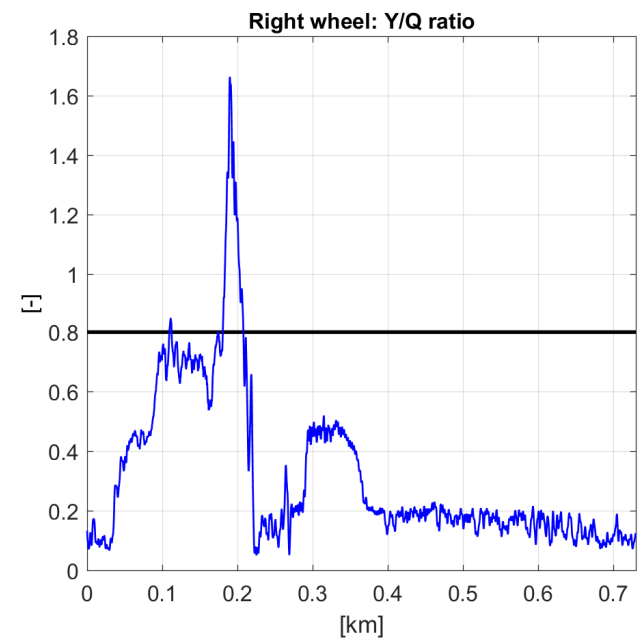

(b)

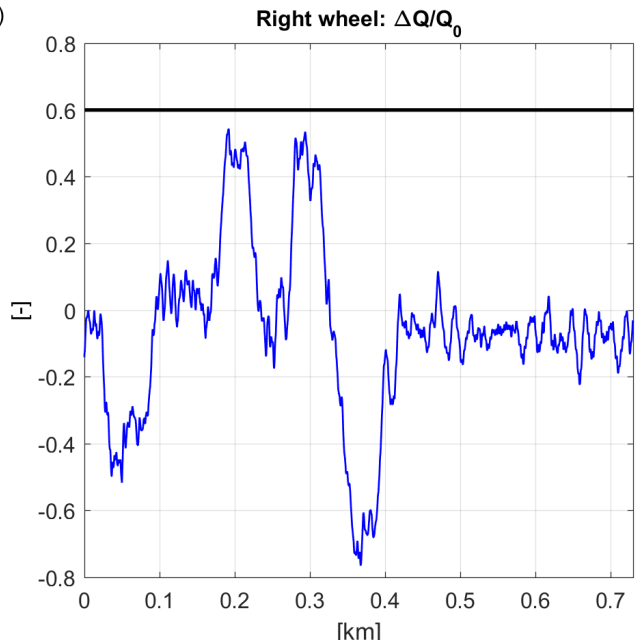

Figure 4: (a) derailment coefficient $\mathrm{Y} / \mathrm{Q}$ and (b) unloading coefficient $\Delta \mathrm{Q} / \mathrm{Q}_{0}$ measured on the right wheel of the instrumented wheelset.

It is important to point out that, although the value of the derailment coefficient is $75 \%$ larger than the limit value given by the Nadal's criterion, the wheel did not show any tendency to flange climb during the test. This experimental result proves that, at least in some running conditions, the Nadal's limit is over-conservative and is not effective in discriminating the actual derailment limit conditions as it does not take into account many factors affecting this limit.

\section{Case study B: vehicle with independently rotating wheels}

This section deals with another example of derailment analysis. Reference is made to a light rail train consisting of three carbodies and three bogies. The two bogies at the vehicle extremities are traditional ones, with motorized solid axles $(71 \mathrm{kN}$ axle load), while the central trailer bogie is equipped with independently rotating wheels (49kN axle load). Experimental tests were carried out in different operating conditions and the leading axle of the central bogie (the most critical one with respect to derailment risk) was instrumented with strain gauges on the spindles, so that the lateral and the vertical contact forces on both wheels could be measured.

Among the various performed tests, that referring to a right curve (900m radius) negotiated at $35 \mathrm{~km} / \mathrm{h}$ is particularly significant. In the curve central portion, the test track included a severe local defect, consisting in an in-phase alignment $(45 \mathrm{~mm})$ and track twist (7\%o) perturbation. The test run was also simulated through a multibody vehicle model particularly appropriate for dynamic analysis of light rail and tram $[18,19]$. Measured data were considered for test track irregularity, wheel/rail profiles (maximum flange contact angle $\left.\gamma_{\max }=73^{\circ}\right)$ and friction coefficient $(\mu=0.5$ ). 


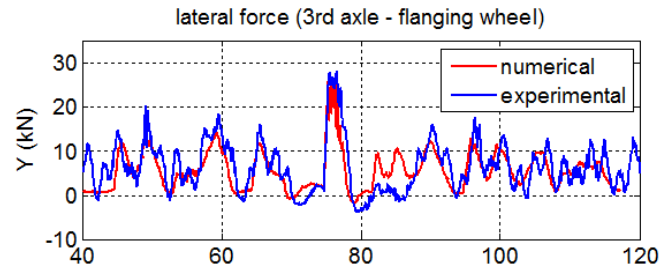

(a)

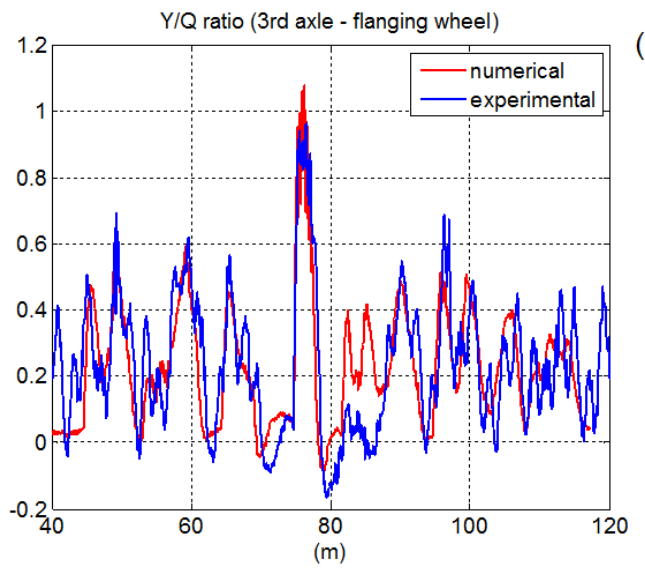

(b)

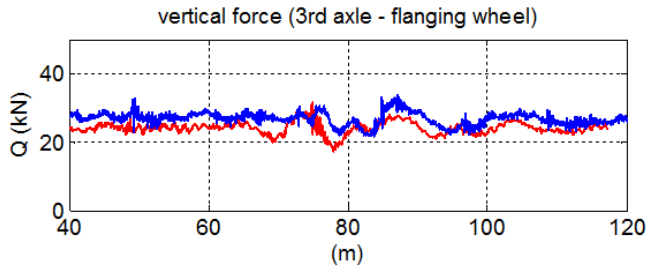

Figure 5: Numerical-experimental comparison: (a) lateral and vertical force on the flanging wheel of the $3^{\text {rd }}$ axle, (b) corresponding Y/Q ratio

Figure 5 shows the results of the numerical-experimental comparison, in terms of forces acting on the flanging wheel of the $3^{\text {rd }}$ axle and corresponding derailment coefficient Y/Q: numerical simulation accurately reproduces the peak in the wheel lateral force which occurs when the vehicle crosses the local track defect, as well as the corresponding maximum value in the Y/Q ratio. Since, according to Nadal's criterion, in the considered derailment analysis $\left(\gamma_{\max }=73^{\circ}, \mu=0.5\right)$, the limit value of $\mathrm{Y} / \mathrm{Q}$ is 1.05 , it can be observed that, during the test of figure 5, a limit condition with respect to derailment risk is reached.

Once validated, the multibody vehicle model can be valuably adopted for simulating flange climb derailment events. Numerical analyses were performed in severe operating conditions, leading to the derailment of the third axle. As an example, Figures 6 and 7 show the numerical results relevant to a 360m-radius left curve, with $0.15 \mathrm{~m}$ superelevation, negotiated at $64 \mathrm{~km} / \mathrm{h}$ : these results refer to the central trailer bogie, equipped with independently rotating wheels. Measured irregularity data were given in input to the simulation.

Figure 6(a) shows the time histories of the calculated wheel/rail relative lateral displacement, for the left and right wheel of the $3^{\text {rd }}$ axle. After curve entrance $(1.5 \mathrm{~s})$, the outer wheel pushes against the rail and, up to $7 \mathrm{~s}$, moves along the curve in flanging condition. At $7 \mathrm{~s}$ the wheel-rail lateral displacement exceeds the maximum gap between the wheelset and the track and derailment occurs. Note that, during curve negotiation, the wheel/rail lateral displacements on the two wheels differ as a result of the elastic deformation of the resilient wheels.

Considering the normal forces on the two contact patches of the flanging wheel (Figure 6(b)), it is possible to observe that in full curve (1.5-7s) contact simultaneously takes place on both the tread and the flange. But what is most worth remarking is that the combined effect of the high centrifugal force and of the track irregularity leads to oscillations in the normal contact forces with increasing amplitude, until derailment is reached. These oscillations are in antiphase on the two contact patches. 

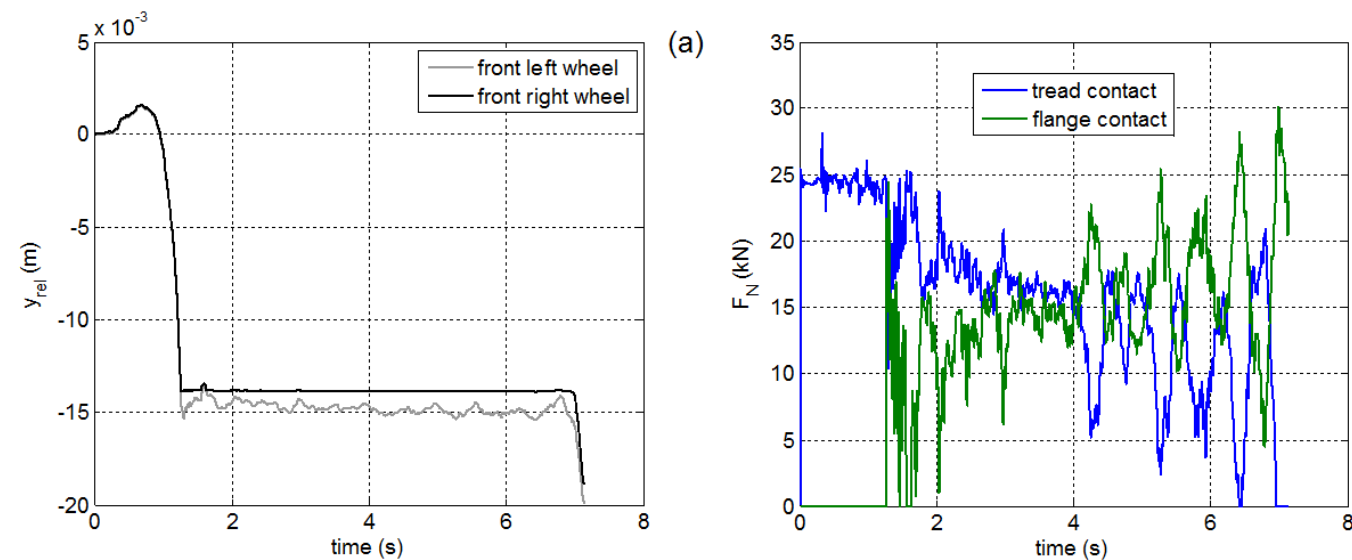

(b)

Figure 6: Derailment simulation (left curve, $\mathrm{R}=360 \mathrm{~m}, 0.15 \mathrm{~m}$ superelevation, $\mathrm{V}=64 \mathrm{~km} / \mathrm{h}$ ): (a) wheel $/$ rail relative lateral displacement, (b) normal contact force on the two contact patches of the flanging wheel

Focussing on the last second of simulation (Figure 7), it can be noticed that contact on the tread is lost a first time around $6.4 \mathrm{~s}$, but then the wheel falls again on the rail. On the contrary, the second loss of contact on the tread, at $7 \mathrm{~s}$, leads to flange climb and to the consequent derailment of the leading outer wheel.
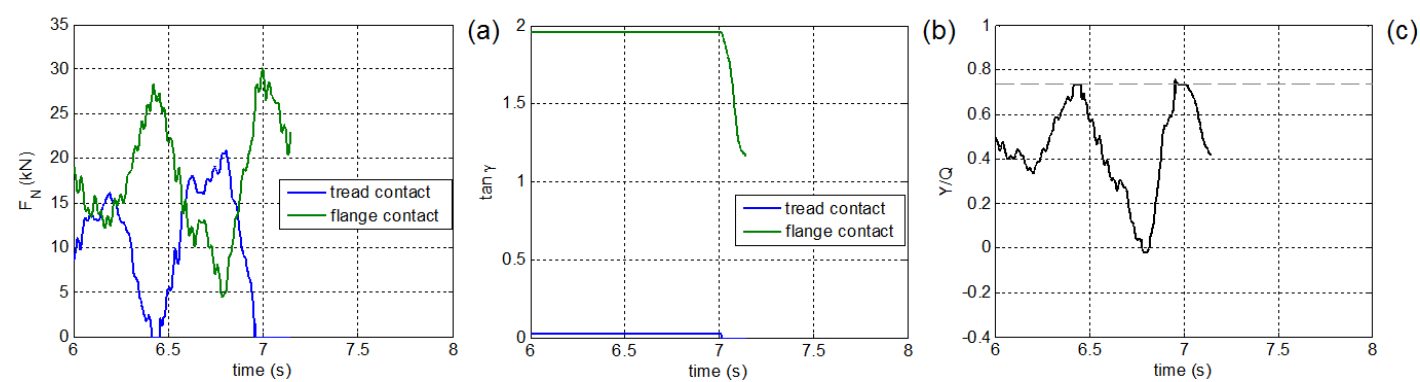

Figure 7: Derailment simulation (left curve, $\mathrm{R}=360 \mathrm{~m}, 0.15 \mathrm{~m}$ superelevation, $\mathrm{V}=64 \mathrm{~km} / \mathrm{h}$ ): (a) normal contact force and (b) contact angle on the two contact patches of the flanging wheel, (c) Y/Q ratio on the same wheel

It is interesting to look at Figure 7(a) together with the time histories of the contact angles on the tread and on the flange (Figure 7(b)). Due to the specific geometrical characteristics of the coupled profiles, as soon as flange contact appears $(1.5 \mathrm{~s})$, the maximum contact angle $\left(\gamma_{\max }=63^{\circ}\right)$ is reached and it then remains constant during the whole curve, until derailment occurs $(7 \mathrm{~s})$. At this time the contact angle suddenly decreases, since the wheel flange is climbing on the rail. Finally, considering the time history of the Y/Q ratio on the flanging wheel (Figure 7(c)), it can be observed that whenever tread contact is lost $(6.4 \mathrm{~s}$ and $7 \mathrm{~s})$ Nadal's limit is reached (it is indicated through the horizontal dashed line positioned at $\mathrm{Y} / \mathrm{Q}=0.74$, which corresponds to $\gamma_{\max }=63^{\circ}$ and $\mu=0.5$ ). This shows that, as expected, Nadal's criterion is definitely appropriate for the detection of derailment risk in the case of 
independently rotating wheels. Whether this danger corresponds or not to actual flange climb (depending on the vehicle dynamic response to track excitation) it is not that important, since safe operation requires proper margin on the Y/Q limit ratio.

The reason why, in the case of a bogie with independently rotating wheels, Nadal's criterion allows to accurately estimate the limit safety condition with respect to derailment is due to the fact that, in this case, the hypotheses of the criterion itself (contact only on the flange, negligible longitudinal creep force, transversal creep force in saturation) are fully satisfied. In particular, the second hypothesis is valid here since we are dealing with independently rotating wheels, with no driving/braking torque applied.

\section{Conclusions}

This paper focused on the analysis of the flange climb specific derailment mechanism. A discussion of the causes that can lead to vehicle derailment was presented, including failures occurring in the running gear (faulty suspensions), bad quality of the infrastructure determining large deviations from the nominal track geometry, or problems occurring at the wheel-rail interface (generating a high coefficient of friction) and extreme environmental conditions. Generally a single factor is not enough to trigger the mechanism but it is necessary to have concurring problems.

Two test cases were presented in this work trying to exemplify these considerations: a vehicle with solid axle and a vehicle with independently rotating wheels. In the former case, the derailment was caused by a combination of a degraded condition of the vehicle and an excessive track twist of the track, while in the latter one the derailment was triggered by large deviations of the track geometry with respect to the nominal one.

The analysis of the State-of-the-Art starting from the famous Nadal's formula proved that many derailment criteria already exist taking into account other important parameters (neglected in Nadal's approach) such as between the vertical forces on the flanging and non-flanging wheels, the wheelset's angle of attack and the magnitude of the longitudinal force.

Anyhow, it is important to point out that these criteria are all based on an approach which considers the flange-climb process as a quasi-static one whereas in some cases, derailment occurs as a consequence of a critical dynamic behaviour of the vehicle caused by large forces applied to the system only for a small duration of time. In these cases, obviously, a quasi-static approach may fall short. For this reason, more research is needed to prove the effectiveness of the existing derailment criteria also in dynamic conditions and/or to propose new criteria fully considering vehicle dynamics.

\section{References}

[1] European Transport Safety Council, "Transport Safety in the EU a Statistical Overview", 2003. 
[2] http://d-rail-project.eu/

[3] N. Wilson, R. Fries, M. Witte, A. Haigermoser, M. Wrang, J. Evans and A. Orlova (2011) Assessment of safety against derailment using simulations and vehicle acceptance tests: a worldwide comparison of state-of-the-art assessment methods, Vehicle System Dynamics, 49:7, 1113-1157.

[4] M.J. Nadal (1896) Théorie de la stabilité des locomotives. Part II: Mouvement de lacet, Ann. Mines 10, pp. 232-255.

[5] J.A. Elkins and H.Wu, New criteria for flange climb derailment, ASME/IEEE Joint Railroad Conference, April 2000.

[6] F. Braghin, S. Bruni and G. Diana (2006) Experimental and numerical investigation on the derailment of a railway wheelset with solid axle, Vehicle System Dynamics, 44:4, 305-325.

[7] Jing Zeng and Qing Hua Guan (2008) Study on flange climb derailment criteria of a railway wheelset, Vehicle System Dynamics, 46:3, 239-251.

[8] J. Santamaria , E. G. Vadillo and J. Gomez (2009) Influence of creep forces on the risk of derailment of railway vehicles, Vehicle System Dynamics, 47:6, 721-752.

[9] R. Spinola Barbosa (2009) Safety of a railway wheelset - derailment simulation with increasing lateral force, Vehicle System Dynamics, 47:12, 1493-1510.

[10] H. Weinstock, Wheel climb derailment criteria for evaluation of rail vehicle safety, Proceedings of the ASME Winter Annual Meeting, New Orleans, 1984, pp. 1-7.

[11] J.A. Elkins and H.Wu (1999) Angle of attack and distance-based criteria for flange climb derailment, Vehicle System Dynamics 33(Suppl.) pp. 291-305.

[12] O. Polach, M. Berg and S. Iwnicki (2006) Chapter 12: Simulation. In: Iwnicki, S. (ed.), Handbook of railway vehicle dynamics. CRC Taylor \& Francis Group, London.

[13] S. Bruni, J. Vinolas, M. Berg, O. Polach, S. Stichel (2011): Modelling of suspension components in a rail vehicle dynamics context, Vehicle System Dynamics, 49:7, 1021-1072.

[14] F. Cheli, R. Corradi, G. Diana, A. Facchinetti, F. Gherardi (2006) Effect of track geometrical defects on running safety of tramcar vehicles, Vehicle System Dynamics, Vehicle System Dynamics 44(Suppl.) pp 302-313.

[15] L. Liang, X. Xin-Biao, J. Xue-Song (2014) Development of a simulation model for dynamic derailment analysis of high-speed trains, Acta Mechanica Sinica 30(6):860-875.

[16] G. Diana, F. Resta, F. Braghin, M. Bocciolone, E. Di Gialleonardo and P. Crosio (2012) Methodology for the calibration of dynamometric wheel-sets for the measurement of the wheel-rail contact forces [Metodologia di calibrazione di sale dinamometriche per la misura delle forze di contatto tra ruota e rotaia], Ingegneria Ferroviaria, 67 (1), pp. 9-21.

[17] UIC (2009) UIC 518: Testing and Approval of Railway Vehicles From the Point of View of Their Dynamic Behaviour - Safety - Track Fatigue - Ride Quality(ed. 4). 
[18] P. Belforte, F. Cheli, R. Corradi, A. Facchinetti (2003) Software for the numerical simulation of tramcar vehicle dynamics, Heavy Vehicle Systems, 10 (1-2), pp. 48-69.

[19] F. Cheli, R. Corradi, G. Diana, A. Facchinetti (2007) Validation of a numerical model for the simulation of tramcar vehicle dynamics by means of comparison with experimental data, Journal of Computational and Nonlinear Dynamics, 2 (4), pp. 299-307. 